\title{
Extreme Deep White Matter Hyperintensity Volumes Are Associated with African American Race
}

\author{
Paul A. Nyquist ${ }^{a-c}$ Murat S. Bilgel ${ }^{d}$ Rebecca Gottesman ${ }^{a}$ Lisa R. Yanek \\ Taryn F. Moy ${ }^{\mathrm{e}}$ Lewis C. Becker ${ }^{\mathrm{e}, \mathrm{f}}$ Jennifer Cuzzocreo ${ }^{\mathrm{d}, \mathrm{g}}$ Jerry Prince ${ }^{\mathrm{d}, \mathrm{g}}$ \\ David M. Yousem ${ }^{g}$ Diane M. Becker ${ }^{e}$ Brian G. Kral ${ }^{e, f}$ Dhananjay Vaidya ${ }^{e}$
}

Departments of a Neurology, ${ }^{b}$ Anesthesiology and Critical Care Medicine, ${ }^{c}$ Neurosurgery and ${ }^{\mathrm{d}}$ Biomedical Engineering and ${ }^{e}$ GeneSTAR Research Program, Division of General Internal Medicine, Department of Medicine, Johns Hopkins School of Medicine, and ${ }^{\mathrm{f}}$ Division of Cardiology, Department of Medicine, and ${ }^{9}$ Divisions of Diagnostic Radiology and Neuroradiology, Department of Radiology, Johns Hopkins Medical Institutions, Baltimore, Md., USA

\section{Key Words}

White matter disease - Women and minorities · Coronary artery disease $\cdot$ Imaging $\cdot$ Risk factors

\begin{abstract}
Background: African Americans (AAs) have a higher prevalence of extreme ischemic white matter hyperintensities (WMHs) on magnetic resonance imaging (MRI) than do European Americans (EAs) based on the Cardiovascular Health Study (CHS) score. Ischemic white matter disease, limited to the deep white matter, may be biologically distinct from disease in other regions and may reflect a previously observed trend toward an increased risk of subcortical lacunar infarcts in AAs. We hypothesized that extreme deep $\mathrm{WMH}$ volume (DWMV) or periventricular volume (PV) may also have a higher prevalence in AAs. Thus, we studied extreme CHS scores and extreme DWMV and PV in a healthy population enriched for cardiovascular disease risk factors. Methods: We imaged the brains of 593 subjects who were first-degree relatives of probands with early onset coronary disease prior to 60 years of age. WMHs were manually delineated on 3-tesla cranial MRI by a trained radiology reader;
\end{abstract}

\section{KARGER}

E-Mail karger@karger.com

www.karger.com/ced the location and volume of lesions were characterized using automated software. DWMV and PV were measured directly with automated software, and the CHS score was determined by a neuroradiologist. Volumes were characterized as being in the upper $25 \%$ versus lower $75 \%$ of total lesion volume. Volumes in the upper versus the remaining quartiles were examined for AA versus EA race using multiple logistic regression (generalized estimating equations adjusted for family relatedness) and adjusted for major vascular disease risk factors including age $\geq 55$ years versus $<55$, sex, current smoking, obesity, hypertension, diabetes and low-density lipoprotein $>160 \mathrm{mg} / \mathrm{dl}$. Results: Participants were $58 \%$ women and $37 \%$ AAs, with a mean age of $51.5 \pm 11.0$ years (range, 29-74 years). AAs had significantly higher odds of having extreme DWMVs (odds ratio, OR, 1.8; 95\% confidence interval, $\mathrm{Cl}, 1.2-2.9 ; \mathrm{p}=0.0076$ ) independently of age, sex, hypertension and all other risk factors. AAs also had significantly higher odds of having extreme CHS scores $\geq 3(\mathrm{OR}, 1.3 ; 95 \% \mathrm{Cl}$, 1.1-3.6; $p=0.025)$. Extreme $P V$ was not significantly associated with $\mathrm{AA}$ race $(\mathrm{OR}, 1.3 ; 95 \% \mathrm{Cl}, 0.81-2.1 ; \mathrm{p}=0.26)$. Con-

B.G.K. and D.V. contributed equally to the paper as senior authors. (c) 2014 S. Karger AG, Basel

$1015-9770 / 14 / 0374-0244 \$ 39.50 / 0$
Paul Nyquist, MD, MPH

Departments of Neurology, Anesthesiology/Critical Care Medicine Neurosurgery, Johns Hopkins School of Medicine 600 N Wolfe Street, Meyer 8-140, Baltimore, MD 21287 (USA) E-Mail pnyquis1@jhmi.edu 
clusions: AAs from families with early-onset cardiovascular disease are more likely to have extreme DWMVs (a subclinical form of cerebrovascular disease) and an extreme CHS score, but not extreme PV, independently of age and other cardiovascular disease risk factors. These findings suggest that this AA population is at an increased risk for DWMV and may be at an increased risk for future subcortical stroke. Longitudinal studies are required to see if DWMV is predictive of symptomatic subcortical strokes in this population.

(c) 2014 S. Karger AG, Basel

\section{Introduction}

Over 790,000 strokes occur annually in the USA, making stroke the fourth leading cause of death and the leading cause of disability in people over the age of 65 [1]. African Americans (AAs) have a higher risk of stroke and subcortical lacunes as well as attendant morbidity and mortality than do European Americans (EAs) $[2,3]$. Ischemic white matter hyperintensities (WMHs) are thought to represent ischemic small vessel disease of the brain and have been associated with stroke and dementia $[4,5]$. The Cardiovascular Health Study (CHS) score is an ordinal measure of WMH that ranges from 0 to 9 [6]. It is based on visual comparison of participant magnetic resonance (MR) images to standardized 'scoring' MR images and represents a qualitative assessment of WMH burden, including periventricular volume (PV) and deep WMH volume (DWMV), as well as ventricular size and atrophy. The Atherosclerosis Risk in Communities (ARIC) study reported that the prevalence of high CHS scores $(\geq 3)$ is greater in AAs than in EAs $[6,7]$. In general, the difference between a CHS of 2 and a CHS of 3 involves a higher burden of subcortical lesion, or DWMV [6]. PV represents the most critical portion of WMH volume measured by the CHS and is more predominant in elderly people than in younger individuals [8-10].

In population-based studies, AAs have been found to have a higher prevalence of subcortical small vessel disease in the form of silent lacunar strokes $[8,11]$. Deep white matter lesions are anatomically specific to the subcortical region of the brain and are thought to have a different pathology and attendant risk factors than WMHs in other regions $[4,12-14]$. It is unknown whether the prevalence of extreme DWMV is greater in AAs than in EAs or if other unique epidemiological characteristics are associated with DWMV. Because of the previously identified association between AA race and subcortical stroke, we hypothesized that subcortically located extreme
DWMV, rather than extreme PV, may be increased in AAs.

To this end we studied the prevalence of severe WMH disease as represented by a CHS score of 3 or greater, as well as extreme DWMV and PV in the upper quartile of the range, in an asymptomatic population of AAs and EAs enriched for vascular risk to determine if extreme WMH was associated with AA race and other risk factors. We applied updated 3.0-tesla magnetic resonance imaging (MRI) volumetrics, which directly quantify and localize DWMV and PV, and compared the data to ordinal CHS scores. Our goal was to determine whether different regions, representing the components of the CHS score, would have different associations with previously identified risk factors, including race [15].

\section{Methods}

\section{Sample and Recruitment}

Participants were recruited from the ongoing prospective study called Genetic Study of Atherosclerosis Risk (GeneSTAR), which was designed to characterize genetic and biological factors associated with incident cardiovascular and cerebrovascular disease in families of patients with early-onset coronary artery disease (CAD). This study was approved by the Johns Hopkins Medicine Institutional Review Board. All participants gave their informed consent. Early-onset CAD was used as a marker for increased familial risk of vascular disease. Probands under the age of $60(39.5 \%$ AA and $33.6 \%$ female) were identified at the time of hospitalization for an early-onset CAD event, including acute myocardial infarction or acute coronary syndromes with angiographic evidence of a flow-limiting stenosis of $>50 \%$ diameter in at least 1 coronary artery. Apparently healthy, asymptomatic siblings and their offspring, and the offspring of the probands, were eligible for this study if they were $29-75$ years of age and had no history of CAD, stroke or transient ischemic attacks. Siblings and offspring were excluded if they had: a history of chronic corticosteroid use; lifethreatening diseases such as active AIDS, renal failure or cancer; neurological diseases that would preclude accurate MRI interpretation, or implanted metals that precluded MRI testing.

\section{Participant Screening}

Subjects underwent comprehensive screening for risk factors. Medical history, current medication use and physical condition were assessed by physical examination and standard methods. Participants self-identified their racial group and were screened for traditional Framingham stroke risk factors, including hypertension, diabetes, smoking and obesity [16]. Participants with atrial fibrillation or symptomatic heart disease were excluded. Anthropometric measures, including height in inches and weight in pounds, were determined with a fixed stadiometer and a balance scale while the participant was wearing light clothing and no shoes. The body mass index was calculated as weight in kilograms divided by height in meters squared. Obesity was defined as a body mass index $\geq 30$, in accordance with the national obesity guidelines [17]. 
Current cigarette smoking was assessed by self-report of any smoking within the past month and/or 2 expired carbon monoxide levels of $\geq 8 \mathrm{ppm}$. Blood pressure was measured 3 times over the course of the day according to American Heart Association guidelines. The average was used to characterize resting blood pressure. Hypertension was defined as an average blood pressure $\geq 140 \mathrm{~mm}$ $\mathrm{Hg}$ systolic or $\geq 90 \mathrm{~mm} \mathrm{Hg}$ diastolic and/or use of an antihypertensive drug. After participants had fasted for 9-12 h overnight, blood was taken for measurement of lipids and glucose. Type 2 diabetes was defined as a physician-diagnosed history, a fasting glucose $\geq 126 \mathrm{mg} / \mathrm{dl}$ and/or use of hypoglycemic antidiabetic medications. Total cholesterol, high-density lipoprotein cholesterol and triglyceride levels were measured according to US Centers for Disease Control standardized methods [18], and low-density lipoprotein cholesterol was estimated by using the Friedewald formula [19]. For persons with triglyceride levels $>400 \mathrm{mg} / \mathrm{dl}$, ultracentrifugation methods were used. Hypercholesterolemia was defined as a low-density lipoprotein cholesterol $\geq 160 \mathrm{mg} / \mathrm{dl}$.

\section{Magnetic Resonance Imaging}

All participants underwent magnetic resonance scanning according to a standard protocol on a Philips 3.0-tesla scanner. The series included the following imaging sequences: (1) axial T1weighted MPRAGE (magnetization prepared rapid gradient echo): TR (repetition time) $10 \mathrm{~ms}$; TE (time to echo) $6 \mathrm{~ms}$; TI (inversion time) voxel size $0.75 \times 0.75 \times 1.0 \mathrm{~mm}^{3}$; contiguous slices, with field of view imaging (FOV) $240 \mathrm{~mm}$; matrix $256 \times 256 \times 160 \mathrm{~mm}$; (2) axial turbo spin echo FLAIR (fluid attenuation inversion recovery): TR 11,000 ms; TI 2,800 ms; TE $68 \mathrm{~ms}$; voxel size $0.47 \times 0.47 \times$ $3.0 \mathrm{~mm}^{3}$; contiguous slices, FOV $240 \mathrm{~mm}$; matrix $256 \times 256 \mathrm{~mm}$. All images were reviewed for clinical pathology, checked, stored first on the in-house reading system and then transferred to an offsite permanent storage facility. Confirmatory clinical reading was completed by a trained neuroradiologist (D.Y.) using the methods of the CHS to define CHS scores on an ordinal scale ranging from 0 to 9 [6]. We considered a CHS score of $>3$ as extreme (provide REF or alternative explanation, e.g. top 15\%). Image processing and volumetric analysis were completed by biomedical engineers and their technical staff.

\section{Volumetric Assessment}

MPRAGE images were skull-stripped and coregistered to FLAIR images. Spatial normalization of the coregistered MPRAGE and FLAIR images into MNI space was performed via affine transformation. A trained rater manually delineated WMHs on the normalized MPRAGE and FLAIR images using Medical Image Processing, Analysis and Visualization software [20]. We segmented the brain in native MPRAGE space using an automated probabilistic methodology that utilizes a topology-preserving algorithm; the resulting tissue mask was mapped to MNI space [21]. We measured total brain intracranial, cortical gray matter, and white matter volumes in native MPRAGE space, and WMH volumes in MNI space. Total brain volume, in cubic millimeters, was identified as the sum of white matter, WMH and gray matter volume from the vertex of the brain to the foramen magnum. Intracranial volume was defined in cubic millimeters as the sum of all dura mater, soft tissue, and sulcal and ventricular cerebrospinal fluid volumes, inferior to bone, from the vertex to the foramen magnum [22].

Spatial characterization of WMHs was carried out with inhouse software designed to determine their location in relation to the ventricles and the deep white matter region in 3-dimensional space. We determined connected components of WMHs with digital 26 connectivity (by measuring all 26 adjacent voxels). We defined periventricular lesions as those that were contiguous with a lesion voxel that was within $4 \mathrm{~mm}$ of the ventricle and defined deep white matter lesions as those that were not contiguous.

\section{Statistical Analysis}

Extreme DWMV and extreme PV were defined as total DWMV or total PV greater than the 75th percentile. Extreme CHS scores were defined as a CHS score of 3 or greater. Demographic and vascular risk factor distributions were tabulated by the presence or absence of extreme CHS score, DWMV and/or PV. To test differences by group, we used t tests for normally distributed variables, Wilcoxon rank sum tests for nonnormally distributed continuous variables and $\chi^{2}$ statistics for categorical variables. The concordance between the dichotomous variables CHS score, extreme DWMV and extreme PV was estimated by using tetrachoric correlation. We used generalized estimating equation regression analyses to correct for intrafamilial correlations and to model being in the highest quartile of DWMV, or PV, or having CHS score $\geq 3$, after adjusting for traditional vascular risk factors, including age, race, sex, hypertension, diabetes, current smoking and obesity.

\section{Results}

\section{Study Sample}

The study population consisted of 593 apparently healthy individuals identified from 324 families of probands with early-onset CAD (1 proband per family). On average, the study population consisted of $1.8 \pm 1.2$ relatives per family (range, 1-8). Siblings of probands comprised $53.1 \%$ of the group, and offspring of siblings and probands comprised $46.9 \%$. Sample characteristics stratified by race are shown in table 1 . Most participants had some white matter disease; $89.9 \%$ had deep white matter disease, $73.7 \%$ had periventricular disease and $14.3 \%$ had a CHS score of 3 or greater.

\section{Association of Extreme DWMV, PV and CHS Score with Race, Controlling for Other Risk Factors}

Results of multivariate regression analyses (generalized estimating equations) to predict extreme $\mathrm{CHS}$ score, extreme DWMV and extreme PV are shown in table 2, respectively. Many of the variables were correlated with one another - DWMV upper quartile and CHS $\geq 3$ : tetrachoric correlation $=-0.3338, \mathrm{p}=0.0868$; $\mathrm{PV}$ upper quartile and CHS $\geq 3$ : tetrachoric correlation $=0.8564, \mathrm{p}=$ 0.0325; DWMV upper quartile and PV upper quartile: tetrachoric correlation $=-0.7485, \mathrm{p}=0.0592$.

Variables independently associated with higher odds of extreme CHS score (table 2) included AA race, older 
Table 1. Demographic characteristics and risk factors of participants by race

\begin{tabular}{lccc}
\hline Characteristic & $\begin{array}{c}\text { AA } \\
(\mathrm{n}=220)\end{array}$ & $\begin{array}{c}\mathrm{EA} \\
(\mathrm{n}=373)\end{array}$ & $\mathrm{p}$ \\
\hline Age, years & $52 \pm 11$ & $52 \pm 10$ & 0.098 \\
Total cholesterol, mg/dl & $192 \pm 43$ & $195 \pm 39$ & 0.38 \\
HDL cholesterol, mg/dl & $58 \pm 16$ & $57 \pm 17$ & 0.36 \\
LDL cholesterol, mg/dl & $115 \pm 39$ & $114 \pm 37$ & 0.69 \\
Diabetes & 21 & 9 & $<0.0001$ \\
Female sex & 64 & 55 & 0.0398 \\
Smoking currently & 24 & 13 & 0.001 \\
Hypertension & 59 & 35 & $<0.0001$ \\
Obesity (BMI $\geq 30)$ & 58 & 39 & $<0.0001$ \\
Hypercholesterolemia & & & \\
$\quad$ (LDL $\geq 160$ mg/dl) & 11 & 10 & 0.58 \\
PV upper quartile & 29 & 23 & 0.087 \\
Total lesion volume upper quartile & 31 & 22 & 0.013 \\
DWMV upper quartile & 33 & 21 & 0.001 \\
\hline
\end{tabular}

Values are means \pm SD or percentages. $\mathrm{BMI}=$ Body mass index; $\mathrm{HDL}=$ high-density lipoprotein; LDL = low-density lipoprotein.

age, current smoking and nonobesity. Variables independently associated with higher odds of extreme DWMV included AA race, older age, female sex and nonobesity (table 2 ). AA race was significantly associated with higher odds of extreme DWMV (OR, 1.8; 95\% CI, $1.2-2.9 ; \mathrm{p}=0.0076$ ), independently of all other risk factors, including hypertension (table 2). When racespecific upper quartiles were used for race-stratified analysis, older age and female sex were associated with higher odds of extreme DWMV in AAs ( $p<0.0001$ and $\mathrm{p}=0.0021$, respectively; table 3 ), whereas older age and thinner body habitus were associated with higher odds of extreme DWMV in EAs ( $<<0.0001$ and $p=0.026$, respectively; table 3 ).

Age was the only variable independently associated with higher odds of extreme PV. AA race was not associated with higher odds of extreme PV (OR, 1.3; 95\% CI, $0.81-2.1 ; \mathrm{p}=0.26$ ), independently of all other risk factors, including hypertension (table 2).

\section{Discussion}

Our results show that the prevalence of extreme DWMV is higher in AA than in EA family members of individuals with premature CAD. This association of AA race with extreme DWMV was independent of age, hypertension and other known cardiovascular disease
Table 2. Fully adjusted logistic regression model predicting CHS $\geq 3$, extreme DWMV and PV

\begin{tabular}{lllc}
\hline Characteristic & OR & $95 \%$ CI & p value \\
\hline CHS $\geq 3$ (n = 593) & & & \\
AA & 1.9 & $1.08-3.5$ & 0.025 \\
Female sex & 1.07 & $0.63-1.8$ & 0.79 \\
Diabetic & 0.73 & $0.34-1.5$ & 0.42 \\
Smoking currently & 2.1 & $0.10-4.2$ & 0.036 \\
Hypertension & 1.7 & $0.99-2.9$ & 0.055 \\
Obesity & 0.56 & $0.32-0.98$ & 0.042 \\
Age (estimate) & 0.14 & $0.11-0.18$ & $<0.0001$ \\
Extreme DWMV (n 593$)$ & & & \\
AA & 1.8 & $1.2-2.9$ & 0.0076 \\
Female sex & 1.6 & $1.1-2.4$ & 0.021 \\
Diabetic & 0.69 & $0.37-1.3$ & 0.22 \\
Smoking currently & 1.3 & $0.77-2.3$ & 0.29 \\
Hypertension & 1.23 & $0.77-2.0$ & 0.39 \\
Obesity & 0.58 & $0.37-0.91$ & 0.18 \\
Age (estimate) & 0.082 & $0.06-0.1$ & $<0.0001$ \\
PV (n = 593) & & & \\
AA & 1.3 & $0.82-2.1$ & 0.26 \\
Female sex & 0.91 & $0.60-1.4$ & 0.66 \\
Diabetic & 1.3 & $0.70-12.3$ & 0.41 \\
Smoking currently & 1.73 & $0.95-3.1$ & 0.068 \\
Hypertension & 0.98 & $0.62-1.6$ & 0.94 \\
Obesity & 0.69 & $0.44-1.07$ & 0.10 \\
Age (estimate) & 0.11 & $0.084-0.13$ & $<0.0001$ \\
\hline
\end{tabular}

$\mathrm{CI}=$ Confidence interval; $\mathrm{OR}=$ odds ratio.

${ }^{1}$ Lesion volume in the upper quartile, with age as a continuous variable.

Table 3. Fully adjusted logistic regression model predicting extreme DWMV in AAs and EAs

\begin{tabular}{llcc}
\hline Characteristic & OR & $95 \% \mathrm{CI}$ & $\begin{array}{l}\mathrm{p} \\
\text { value }\end{array}$ \\
& & & \\
\hline In AAs (n=220) & & & \\
$\quad$ Female sex & 2.7 & $1.4-5.3$ & 0.0021 \\
Diabetic & 0.54 & $0.26-1.14$ & 0.11 \\
Smoking currently & 1.7 & $0.76-3.8$ & 0.20 \\
Hypertension & 1.3 & $0.59-2.7$ & 0.56 \\
Obesity & 0.64 & $0.33-1.23$ & 0.18 \\
Age (estimate) & 0.084 & $0.048-0.12$ & $<0.0001$ \\
In EAs (n = 373) & & & \\
Female sex & 1.1 & $0.67-1.9$ & 0.68 \\
Diabetic & 1.1 & $0.43-2.7$ & 0.87 \\
Smoking currently & 1.1 & $0.48-2.5$ & 0.83 \\
Hypertension & 1.2 & $0.65-2.2$ & 0.55 \\
Obesity & 0.47 & $0.24-0.91$ & 0.026 \\
Age (estimate) & 0.082 & $0.054-0.11$ & $<0.0001$ \\
\hline
\end{tabular}

$\mathrm{CI}=$ Confidence interval; $\mathrm{OR}=$ odds ratio. Lesion volumes as found in the upper quartile, with age as a continuous variable. 
risk factors. This study confirms the observations of the previous ARIC study, which found an association between AA race and extreme CHS score [7]. Additionally, it builds on the results of the ARIC study by using modern, validated, direct measurements of DWMV with automated white matter segmentation. These methods allow for the attribution of lesions to a brain region and analysis of associations between different risk factors and regions.

AA race has been associated with an increased prevalence of many vascular disease phenotypes, including symptomatic and asymptomatic subcortical lacunar stroke and vascular disease in other organ systems, such as the coronary arteries and peripheral vasculature [2329]. Furthermore, racial differences in white matter disease burden have been reported to be related to smoking and increased rates and severity of hypertension $[7,30]$. Although other studies have emphasized smoking as a risk factor, in our study, smoking was not associated with extreme DWMV in the stratified or combined analysis. Likewise, hypertension did not appear to drive the association between DWMV and AA race. Our analysis of DWMV predictors controlled for the diagnosis of hypertension as well as age. However, we cannot exclude the possibility that genetic differences that affect hypertension, such as angiotensin-converting enzyme polymorphisms, might be more prevalent in this AA population [9]. Additionally, it is possible that an unidentified inheritable trait may be associated with the AA population in this study of related individuals.

Many of our observed associations and correlations support the idea that extreme DWMV is an independent lesion type that conveys risks that differ from those of PV and CHS score. Extreme PV and extreme CHS score shared similar associations, including age, smoking and decreased obesity. It is important to note that hypertension in AAs may represent an undertreated disease with an earlier age of onset as compared to EAs which could have contributed to the increased predominance of extreme DWMV in this group. We were unable to designate the age of onset and control for this factor in our analysis. The correlation between CHS score and PV was stronger than the correlation between CHS score and DWMV. Extreme DWMV was associated with AA race, as was extreme CHS score. However, in our stratified analysis, DWMV was independently associated with female sex but lacked associations with smoking, hypertension and obesity, which were associated with extreme CHS score, despite the fact that the CHS scale emphasized periventricular confluence. In the higher grades of CHS score ( $>6)$, involvement of the centrum semiovale is emphasized. These observations would support those of Fazekas et al. [31], who reported different pathological substrates in the periventricular and DWMV regions. In particular we did include the PV caps into the PV calculation. Lesions in this region are reported to be nonischemic in nature. Thus, the lower PV relative to DWMV may reflect a proclivity for ischemic lesions and higher extreme DWMV as compared to PV.

Small vessel vascular disease in the subcortical region has been reported to be more prevalent in the AA population than in either the EA or Hispanic population [2329]. The DWMV and periventricular lesions lie in the subcortical and periventricular regions, respectively. These regions have very different small vessels, with long cortically based small vessels serving the periventricular region and shorter small vessel perforators serving the subcortical DWMV. In past studies, different locations of $\mathrm{WMH}$ have been associated with different risk factors and clinical outcomes $[32,33]$. Investigators who have analyzed the pathological nature of deep white matter lesions have reported that risk factors such as endothelial activation and inflammation are more prominent in the short subcortical vessels of the deep white matter than in the long periventricular vessels $[4,12,32,34]$. Our observations may result from enrichment of these risk factors in our study population [35].

Our findings have similarities to those of the ARIC study of severe CHS scores [7]. In both studies, extreme CHS score was associated with AA race, as well as age, hypertension and smoking. The association of extreme CHS with smoking and hypertension appeared to be stronger in AAs than in EAs in both studies. However, our population differed from that in the ARIC study. Our population was by definition asymptomatic, whereas the ARIC group included both symptomatic and asymptomatic individuals. All of our participants were relatives of a proband, whereas for the most part, ARIC participants were unrelated. The average age in our population was lower - 52, as compared to 62 in the ARIC cohort - and unlike the ARIC population, our population was notably enriched with vascular risk factors $[6,35]$. Interestingly, however, the percentage of participants with extreme CHS scores was very similar in the two studies: $14.7 \%$ in our population and $12.5 \%$ in the ARIC study [36]. Our study is unusual in that obesity appeared to be associated with a reduced risk of $\mathrm{WMH}$ of all types by all measures. Although this finding has been reported in other studies, such as the Woman's 
Health Initiative MRI Study [37], in the preponderance of the literature, an increased body mass index is strongly associated with an increased risk of WMH and WMH progression $[5,16,18,19,24,38,39]$. As shown in other studies, age was the predominant characteristic associated with WMHs of all types [40].

Other unique aspects of our study include the use of an MRI scanner with a 3.0-tesla field strength. This MRI scanner has a greater sensitivity for the detection of WMHs than does the 1.5-tesla scanner and provides better assessment of lesion volume through improved signal-to-noise ratios $[13,15]$. In general, prevalence and volume measured for deep white matter lesions and periventricular lesions were significantly higher than those obtained with similar 1.5-tesla methodologies [10]. We also used advanced programming that has been extensively validated to separate, localize and measure $\mathrm{WMH}$ volumes. This program has also been extensively validated in other white matter diseases such as multiple sclerosis [20].

\section{Conclusion}

AA race is an independent risk factor for extreme DWMV and extreme CHS scores in a population enriched for vascular risk factors. In contrast, PV is not associated with AA race. Extreme DWMV had unique epidemiological associations, suggesting that it represents a unique lesion type that differs from PV lesions. The association between extreme DWMV, which is a subclinical form of ischemic stroke, and AA race suggests that this population may have an increased risk of future subcortical stroke. This risk must be verified in future longitudinal studies.

\section{Acknowledgments}

Research reported in this publication was supported by the $\mathrm{Na}$ tional Institute of Neurological Disorders and Stroke of the National Institutes of Health under Award No. R01NS062059. Claire F. Levine, MS, ELS, contributed to the editing of this manuscript.

\section{References}

1 Lloyd-Jones D, Adams R, Carnethon M, De Simone G, Ferguson TB, Flegal K, Ford E, Furie K, Go A, Greenlund K, Haase N, Hailpern S, Ho M, Howard V, Kissela B, Kittner S, Lackland D, Lisabeth L, Marelli A, McDermott M, Meigs J, Mozaffarian D, Nichol G, O’Donnell C, Roger V, Rosamond W, Sacco R, Sorlie P, Stafford R, Steinberger J, Thom T, Wasserthiel-Smoller S, Wong N, Wylie-Rosett J, Hong Y; American Heart Association Statistics Committee, Stroke Statistics Subcommittee: Heart disease and stroke statistics - 2009 update: a report from the American Heart Association Statistics Committee and Stroke Statistics Subcommittee. Circulation 2009;119:480-486.

$\checkmark 2$ Gorelick PB: Cerebrovascular disease in African Americans. Stroke 1998;29:26562664.

3 White H, Boden-Albala B, Wang C, Elkind MS, Rundek T, Wright CB, Sacco RL: Ischemic stroke subtype incidence among whites, blacks, and Hispanics: the Northern Manhattan Study. Circulation 2005;111:13271331.

4 Young VG, Halliday GM, Kril JJ: Neuropathologic correlates of white matter hyperintensities. Neurology 2008;71:804-811.

5 Vermeer SE, Hollander M, van Dijk EJ, Hofman A, Koudstaal PJ, Breteler MM: Silent brain infarcts and white matter lesions increase stroke risk in the general population: the Rotterdam Scan Study. Stroke 2003;34: 1126-1129.
6 Manolio TA, Kronmal RA, Burke GL, Poirier V, O'Leary DH, Gardin JM, Fried LP, Steinberg EP, Bryan RN: Magnetic resonance abnormalities and cardiovascular disease in older adults. The Cardiovascular Health Study. Stroke 1994;25:318-327.

7 Liao D, Cooper L, Cai J, Toole J, Bryan N, Burke G, Shahar E, Nieto J, Mosley T, Heiss G: The prevalence and severity of white matter lesions, their relationship with age, ethnicity, gender, and cardiovascular disease risk factors: the ARIC Study. Neuroepidemiology 1997;16:149-162.

8 Sacco RL, Kargman DE, Gu Q, Zamanillo MC: Race-ethnicity and determinants of intracranial atherosclerotic cerebral infarction. The Northern Manhattan Stroke Study. Stroke 1995;26:14-20.

9 Raz N, Yang Y, Dahle CL, Land S: Volume of white matter hyperintensities in healthy adults: contribution of age, vascular risk factors, and inflammation-related genetic variants. Biochim Biophys Acta 2012;1822:361369.

10 Wen W, Sachdev P: The topography of white matter hyperintensities on brain MRI in healthy 60 - to 64 -year-old individuals. Neuroimage 2004;22:144-154.

11 Prabhakaran S, Wright CB, Yoshita M, Delapaz R, Brown T, DeCarli C, Sacco RL: Prevalence and determinants of subclinical brain infarction: the Northern Manhattan Study. Neurology 2008;70:425-430.
12 Fernando MS, Simpson JE, Matthews F, Brayne C, Lewis CE, Barber R, Kalaria RN, Forster G, Esteves F, Wharton SB, Shaw PJ, O'Brien JT, Ince PG: White matter lesions in an unselected cohort of the elderly: molecular pathology suggests origin from chronic hypoperfusion injury. Stroke 2006;37:1391-1398.

13 Jack CR Jr, O'Brien PC, Rettman DW, Shiung MM, Xu Y, Muthupillai R, Manduca A, Avula R, Erickson BJ: FLAIR histogram segmentation for measurement of leukoaraiosis volume. J Magn Reson Imaging 2001;14:668-676.

-14 Ramirez J, Gibson E, Quddus A, Lobaugh NJ, Feinstein A, Levine B, Scott CJ, Levy-Cooperman N, Gao FQ, Black SE: Lesion Explorer: a comprehensive segmentation and parcellation package to obtain regional volumetrics for subcortical hyperintensities and intracranial tissue. Neuroimage 2011;54:963-973.

15 Van den Heuvel DM, ten Dam VH, de Craen AJ, Admiraal-Behloul F, van Es AC, Palm WM, Spilt A, Bollen EL, Blauw GJ, Launer L, Westendorp RG, van Buchem MA; PROSPER Study Group: Measuring longitudinal white matter changes: comparison of a visual rating scale with a volumetric measurement. AJNR Am J Neuroradiol 2006;27:875-878.

-16 Wang TJ, Massaro JM, Levy D, Vasan RS, Wolf PA, D'Agostino RB, Larson MG, Kannel WB, Benjamin EJ: A risk score for predicting stroke or death in individuals with new-onset atrial fibrillation in the community: the Framingham Heart Study. JAMA 2003;290:10491056.
Extreme DWMVs and African American Race
Cerebrovasc Dis 2014;37:244-250 DOI: $10.1159 / 000358117$ 


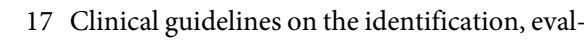
uation, and treatment of overweight and obesity in adults: executive summary. Expert Panel on the Identification, Evaluation, and Treatment of Overweight in Adults. Am J Clin Nutr 1998;68:899-917.

18 Myers GL, Kimberly MM, Waymack PP, Smith SJ, Cooper GR, Sampson EJ: A reference method laboratory network for cholesterol: a model for standardization and improvement of clinical laboratory measurements. Clin Chem 2000;46:1762-1772.

19 Tullberg M, Fletcher E, DeCarli C, Mungas D, Reed BR, Harvey DJ, Weiner MW, Chui HC, Jagust WJ: White matter lesions impair frontal lobe function regardless of their location. Neurology 2004;63:246-253.

20 Bazin PL, Cuzzocreo JL, Yassa MA, Gandler W, McAuliffe MJ, Bassett SS, Pham DL: Volumetric neuroimage analysis extensions for the MIPAV software package. J Neurosci Methods 2007;165:111-121.

21 Shiee N, Bazin PL, Ozturk A, Reich DS, Calabresi PA, Pham DL: A topology-preserving approach to the segmentation of brain images with multiple sclerosis lesions. Neuroimage 2010;49:1524-1535.

22 Carass A, Cuzzocreo J, Wheeler MB, Bazin PL, Resnick SM, Prince JL: Simple paradigm for extra-cerebral tissue removal: algorithm and analysis. Neuroimage 2011;56:19821992.

-23 Kobayashi S, Okada K, Koide H, Bokura H, Yamaguchi S: Subcortical silent brain infarction as a risk factor for clinical stroke. Stroke 1997;28:1932-1939.

-24 Uehara T, Tabuchi M, Mori E: Risk factors for silent cerebral infarcts in subcortical white matter and basal ganglia. Stroke 1999;30:378382.

25 Ohira T, Shahar E, Chambless LE, Rosamond WD, Mosley TH Jr, Folsom AR: Risk factors for ischemic stroke subtypes: the Atherosclerosis Risk in Communities study. Stroke 2006; 37:2493-2498.
26 Rosamond W, Broda G, Kawalec E, Rywik S, Pajak A, Cooper L, Chambless L: Comparison of medical care and survival of hospitalized patients with acute myocardial infarction in Poland and the United States. Am J Cardiol 1999;83:1180-1185.

27 Mensah GA, Mokdad AH, Ford ES, Greenlund KJ, Croft JB: State of disparities in cardiovascular health in the United States. Circulation 2005;111:1233-1241.

28 Feinstein M, Ning H, Kang J, Bertoni A, Carnethon M, Lloyd-Jones DM: Racial differences in risks for first cardiovascular events and noncardiovascular death: the Atherosclerosis Risk in Communities study, the Cardiovascular Health Study, and the Multi-Ethnic Study of Atherosclerosis. Circulation 2012;126:5059.

29 Hozawa A, Folsom AR, Sharrett AR, Chambless LE: Absolute and attributable risks of cardiovascular disease incidence in relation to optimal and borderline risk factors: comparison of African American with white subjects - Atherosclerosis Risk in Communities Study. Arch Intern Med 2007;167:573-579.

30 Gottesman RF, Coresh J, Catellier DJ, Sharrett AR, Rose KM, Coker LH, Shibata DK, Knopman DS, Jack CR, Mosley TH Jr: Blood pressure and white-matter disease progression in a biethnic cohort: Atherosclerosis Risk in Communities (ARIC) study. Stroke 2010; 41:3-8.

Fazekas $F$, Kleinert $R$, Offenbacher $H$, Schmidt R, Kleinert G, Payer F, Radner H, Lechner H: Pathologic correlates of incidental MRI white matter signal hyperintensities. Neurology 1993;43:1683-1689.

32 Rostrup E, Gouw AA, Vrenken H, van Straaten EC, Ropele S, Pantoni L, Inzitari D, Barkhof F, Waldemar G; LADIS Study Group: The spatial distribution of age-related white matter changes as a function of vascular risk factors - results from the LADIS study. Neuroimage 2012;60:1597-1607.
33 Enzinger C, Smith S, Fazekas F, Drevin G, Ropele S, Nichols T, Behrens T, Schmidt R, Matthews PM: Lesion probability maps of white matter hyperintensities in elderly individuals: results of the Austrian stroke prevention study. J Neurol 2006;253:1064-1070.

34 Hassan A, Hunt BJ, O’Sullivan M, Parmar K, Bamford JM, Briley D, Brown MM, Thomas DJ, Markus HS: Markers of endothelial dysfunction in lacunar infarction and ischaemic leukoaraiosis. Brain 2003;126:424-432.

35 Becker DM, Yook RM, Moy TF, Blumenthal RS, Becker LC: Markedly high prevalence of coronary risk factors in apparently healthy African-American and white siblings of persons with premature coronary heart disease. Am J Cardiol 1998;82:1046-1051.

- 36 Simoni M, Li L, Paul NL, Gruter BE, Schulz UG, Kuker W, Rothwell PM: Age- and sexspecific rates of leukoaraiosis in TIA and stroke patients: population-based study. Neurology 2012;79:1215-1222.

37 Goveas JS, Espeland MA, Hogan P, Dotson V, Tarima S, Coker LH, Ockene J, Brunner R, Woods NF, Wassertheil-Smoller S, Kotchen JM, Resnick S: Depressive symptoms, brain volumes and subclinical cerebrovascular disease in postmenopausal women: the Women's Health Initiative MRI Study. J Affect Disord 2011;132:275-284

38 Putaala J, Haapaniemi E, Kurkinen M, Salonen O, Kaste M, Tatlisumak T: Silent brain infarcts, leukoaraiosis, and long-term prognosis in young ischemic stroke patients. Neurology 2011;76:1742-1749.

- 39 Arsava EM, Bayrlee A, Vangel M, Rost NS Rosand J, Furie KL, Sorensen AG, Ay H: Severity of leukoaraiosis determines clinical phenotype after brain infarction. Neurology 2011;77:55-61.

40 Jeerakathil T, Wolf PA, Beiser A, Massaro J, Seshadri S, D’Agostino RB, DeCarli C: Stroke risk profile predicts white matter hyperintensity volume: the Framingham Study. Stroke 2004;35:1857-1861. 